\title{
Identification of heart disease-prone personality using oscillometric blood pressure measurements
}

\author{
Seungah Jung ${ }^{\mathrm{a}}$ and Youngsuk Shin ${ }^{\mathrm{b}, *}$ \\ ${ }^{a}$ Department of Counseling Psychology, Chosun University, Gwangju, Korea \\ ${ }^{\mathrm{b}}$ Department of Information and Communication Engineering, Chosun University, Gwangju, Korea
}

\begin{abstract}
.
BACKGROUND: By using the conventional method of measuring two-points (systolic and diastolic) blood pressure, it is difficult to differentiate a heart disease-prone personality from normals. Recently, an oscillometric method that reflects the personalized trait of blood pressure was developed by one author.

OBJECTIVE: By using this new measurement technique, this study intended to test the possibility of differentiating a heart disease-prone personality (type A or type D) from normal people.

METHODS: TPA scale in MMPI-2 and DS14 were used for screening type A and D. Oscillometric waveforms created by the cuff pressure were segmented into window blocks based on a single beat at a minimum, allowing this method to extract maximal top and bottom amplitudes in each window block. Then, a spectrogram using a short-time Fourier transform was applied to discriminate between character types in extracted blood pressure patterns with linear discriminant analysis.

RESULTS: Compared to a normal personality, type A and type D personalities displayed a lower frequency response on STFT with maximum negative amplitudes than normals. In particular, the type $\mathrm{D}$ personality showed a lower frequency response than the type A personality.

CONCLUSION: These results could provide a new qualitative method for measuring different biological indices between type A or D personalities and normals.
\end{abstract}

Keywords: Blood pressure oscillation pattern, cardiovascular disease, a short-time Fourier transform, type A, type D personality

\section{Introduction}

Personality is a main factor that influences personal behavior. Personality types also affect disease behavior and may cause certain physical diseases. This view has become known in the fields of health psychology and behavioral medicine. In particular, type A and type D personalities have been suggested as risk factors for certain physical diseases (coronary disease) [1]2]. In 1959, cardiologists Meyer Friedman and Ray Rosenman first described and defined the type A personality. In the beginning 1970s, collaborating with a group of psychologists, Friedman also followed 1013 heart attack survivors for four and a half years to determine the effects of altering their coronary-prone (type A) behavior patterns. Results indicated that behavioral counseling reduced rates of recurrence from $21 \%$ (or higher) to

\footnotetext{
${ }^{*}$ Corresponding author: Youngsuk Shin, Department of Information and Communication Engineering, Chosun University, \#309 Pilmundaero, Dong-gu, Gwangju 501-759, Korea. Tel.: +82 62230 7011; Fax: +82 62230 7011; E-mail: ysshin@ chosun.ac.kr.
} 
$13 \%$ [3]. However, some later studies reported inconsistent results in the relationship between type A and coronary heart disease. Other scholars insisted that the structural concept of the type A personality was mixed with heterogeneous personality characteristics. Simultaneously, studies appeared regarding the opposite relationship between the type A personality and coronary heart disease (CHD), and more specific studies of personality types causing CHD followed [4-7]. Johan Denollet, professor of medical psychology at Tilburg University, proposed the construct of the type D personality based on clinical observations in cardiac patients, empirical evidence, and existing theories of personality. Denollet defined type D personality as the joint tendency toward negative affectivity (e.g., worry, irritability, gloom) and social inhibition. Recently, a scale for measuring the type D personality character was developed and became widely used [8].

Until now, many researches related to cardiovascular disease have been done the significance of automated detection and prediction of cardiac abnormalities. Many studies analyze heart rate variability signals to detect abnormal rhythms of heart. Some cases of these automatic arrhythmia detection and classification techniques are neural network [9], support vector machines [10], and genetic programming [1112]. The proposed method in this paper studies pulse waves signals of normal episodes out of arrhythmia. While personality traits can be a risk factor for CHD, hypertension is traditionally recognized as a physical risk factor that affects cardiovascular disease. The rate of hypertension in Korea reported by MHW (Ministry of Health and Welfare of Korea) in 2014 [13] increased gradually from $24.6 \%$ in 2007 to $29.0 \%$ in 2012 . The prehypertension rate for people in their thirties was $27.3 \%$ (standard error of $1.7 \%$ ). Those in their forties showed a prehypertension rate of $27.6 \%$ (standard error of $1.9 \%$ ). The measuring of blood pressure is a popular method that diagnoses the hypertension. Many studies of blood pressure measurement have used the oscillometric method. The oscillometric method measures the oscillation amplitudes generating from a sequentially deflating cuff to determine blood vessel systolic and diastolic pressures. The systolic pressure is the maximum pressure on the arteries, which occurs when the heart contracts and pushes blood through the arteries. On the other hand, the diastolic pressure is the pressure in the arteries, which occurs when the heart's left ventricle rests between beats [14[15]. Compared to a more invasive technique, this measurement method is simple and allows patients to feel comfortable. However, this method of measuring two points blood pressure has an important weakness: it is difficult to detect personalized pattern or the qualitative characteristics of blood-pressure.

Recently, one of the authors developed oscillometric method that reflects the personalized traits of blood pressure [16 17]. This newly developed oscillometric blood pressure measurement method extracts maximum positive amplitude (MPA) and maximum negative amplitude (MNA) from the oscillation amplitudes in the divided feature windows. One segmented feature window is set at a minimum, based on a single beat in the sample window. The MPA and MNA reflect the variances in the systolic and diastolic blood pressures. MPA is the amplitude of the maximal top pulse in the divided one-feature window. MNA is the amplitude of the maximal bottom pulse in divided one-feature window. The oscillation amplitude pattern extracted from segmented window blocks reflects an individual's characteristics such as physiological traits, hypertension, gender, and age. The research results indicated that the extracted features display a fairly uniform pattern for each age group. In particular, prehypertensive subjects ranging from 10 years to 30 years old exhibited lower amplitudes than subjects ranging from 40 years to 80 years old in one-third section of the feature windows [16].

This study explores whether the character of type A and type D personalities can be distinguished from the character of a normal type personality in an age group of individuals in their twenties, using a newly developed oscillometric blood pressure measurement method. If this study shows that the character of 


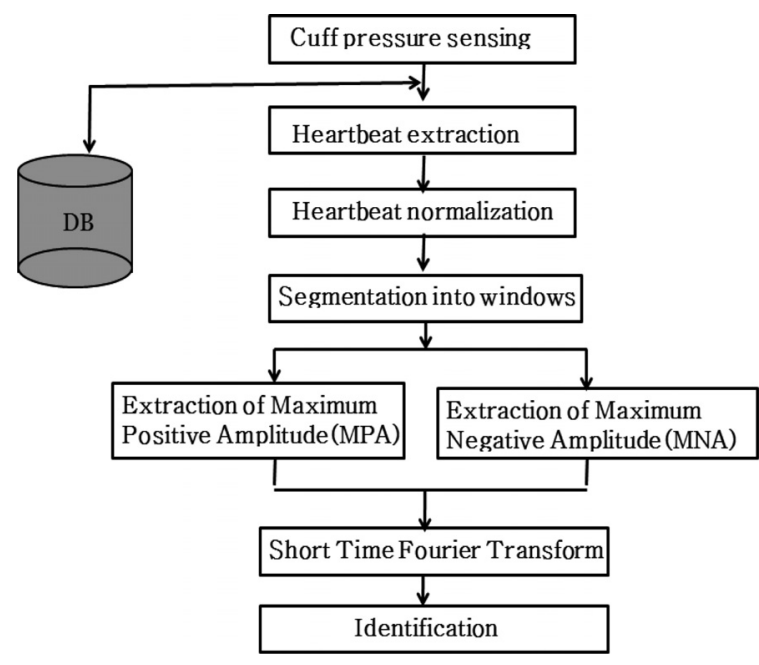

Fig. 1. Flow diagram applied to this study.

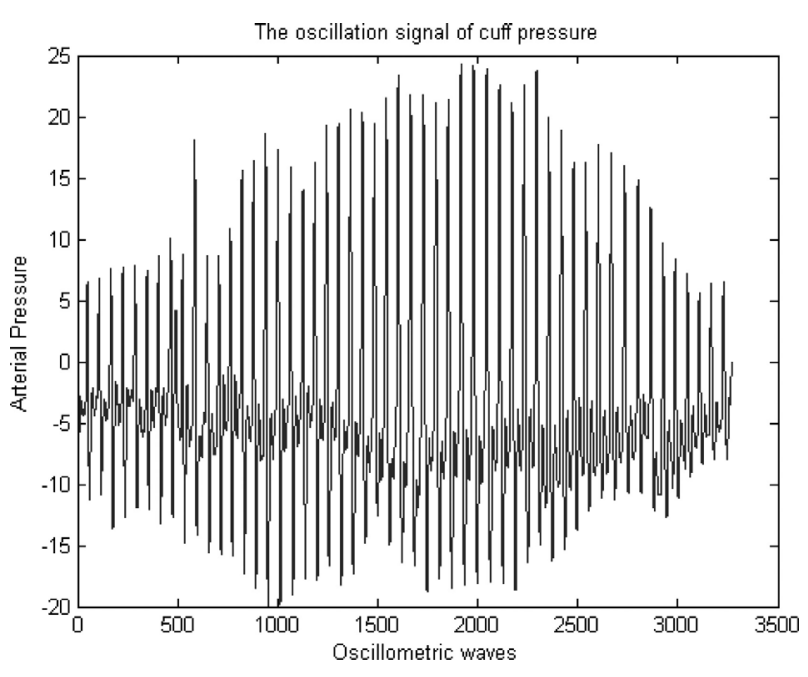

Fig. 2. Blood pressure oscillation pattern of one subject, extracted by the cuff pressure using the oscillometric method.

type A and type D personalities can be differentiated from the character of the normal type personality, these results can be used as verification data to examine the correlation between personality type and cardiovascular diseases in early age groups. In addition, personality identification through blood pressure patterns may help prevent potential cardiovascular disease.

\section{Methodology}

In this section, we explain a feature extracting method and classification algorithm that can discriminate the character of type $\mathrm{A}$ and type $\mathrm{D}$ personalities from the character of the normal type personality. To do this, we used newly develop method of measuring oscillometric blood pressure pattern. We performed this work in four steps. First step described the process of data collection. Second step specified the method of preprocessing to make the oscillation waves into constant length. Third step developed a method of representing the character of type A and type D personalities via oscillation amplitude features from the uniformed oscillation waves. Finally, we classified the represented features into three personality types using a linear discriminant analysis algorithm. Figure 1 shows a flow diagram applied to this study.

\subsection{Data collection}

This study was approved by the Human Ethics Research Committee of Chosun University prior to data collection. The experimental participants consisted of 67 (45\%) male and 81 (55\%) female college students, whose mean age was 21.6 (SD 3.8 years). When the participants reported having been any heart related disease or taking any related medicine, they were excluded from this study at the first. And all the participants were informed beforehand that they should not take any stimulants or sedatives such as coffee, sleeping pills prior to measuring their blood pressure. The database consists of the pressure oscillation data from the oscillometric method and the discrimination data between type A and type D by the self-reporting method. 
Individual blood pressure patterns of the experimental participants were obtained from pressure oscillation data that was extracted by oscillometric method. Participants were guided to relax in a waiting room for 10 minutes, and the waiting room is a pre-organized measurement room where the blood pressure could be measured accurately. Three recordings per subject were taken from 148 male and female participants and each measuring process was repeated after each one-minute rest time. The three-time measurement was used for the following reason: the average difference value of measurement between published study that used five records [16] and ours that used three records were negligible (0.0001). This result reflects that our new method is not sensitive to the number of measuring times. Through these processes, we obtained a total of 444 records per subject. Figure 2 shows a sample of an oscillation pattern obtained using the oscillometric measurement method on one subject.

The discrimination between type A and type D group was examined using a type A scale (TPA) in MMPI-2 (Minnesota Multiphasic Personality Inventory-2), and a type D scale (DS14). The TPA scale [18] is one of the content scales in MMPI-2, which is constructed according to Freedman's concept of the type A personality. In this study, subjects were defined as type A personalities when they received scores higher than $60 \mathrm{~T}$ on the TPA scale. When we adopted this criterion, 12 subjects $(8.1 \%)$ were classified into the type A group. The type D personality was measured using DS14, a Korean version of the original Denollet's scale [19]. According to Denollet, type D is assigned when scores of two subscales of DS14 (NE: negative emotionality and SI: social inhibition) are both above 10. In our sample, 46 students $(31.1 \%)$ were identified as having a type D personality. Eighty-six students $(58.1 \%)$ were classified as normal because they did not met at any criterion of type A or type D personalities by the TPA scales and DS14. We excluded four subjects (2.7\%) who met the criteria for both type A and type D from this study.

\subsection{Preprocessing}

Oscillation waveforms generated by cuff have an oscillation pattern that varies in size over time for a given subject. A similar number of oscillation waveforms could be observed in three measurements of the same subject. For this reason, we attempted normalization process to minimize variations of oscillation waveforms of the same subject. The applied normalization used the existing study method [16]. Given the training set $X=\left\{X_{i}\right\}_{i=1}^{N}$, containing $N$ oscillation patterns $X_{i}=\left\{X_{i j}\right\}_{j=1}^{N_{i}}$, where each oscillation pattern $X_{i j}$ consists of a number of oscillation waveforms. The normalization is applied to the following Eq. (1):

$$
\omega=\sqrt{\sum_{j=1}^{N_{i}}\left(X_{i j}\right)^{2}}, X_{i}^{*}=\frac{X_{i}}{\omega} .
$$

\subsection{Feature extraction}

To extract the personalized trait that distinguishes one person's blood pressure pattern from the others, this study divides a normalized oscillation pattern into windows. We set the criterion of this segmentation that at least one heart beat should be included in the minimal oscillation waveforms. The number of windows is defined using two parameters. The parameter $\gamma$ describes the minimal number of oscillation waveforms in the entire blood pressure pattern and $\delta$ specifies the number of oscillation waveforms 


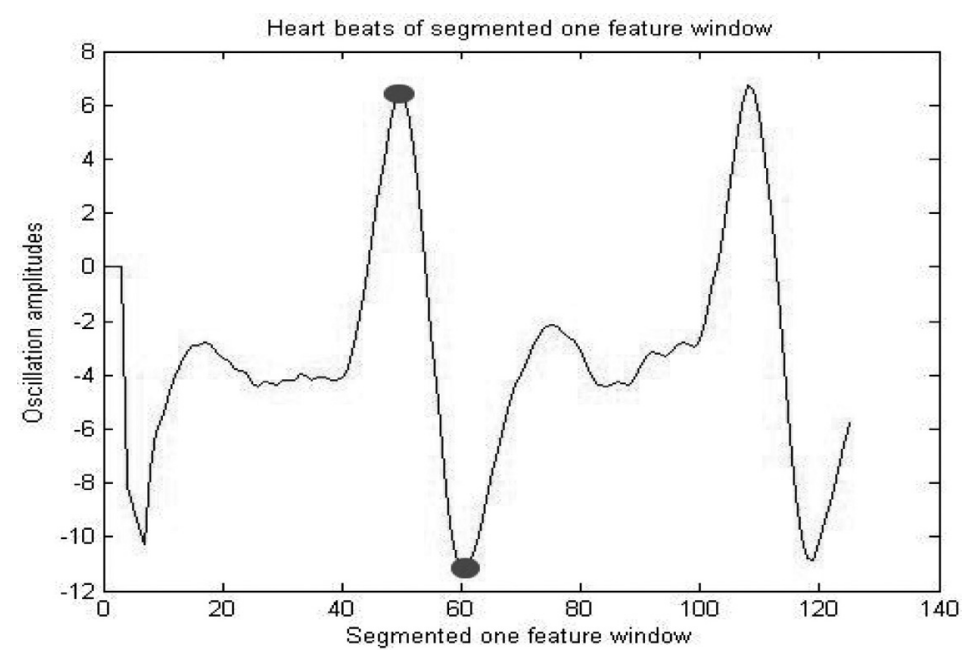

Fig. 3. Two heartbeats from one subject, detected in the segmented one-feature window.

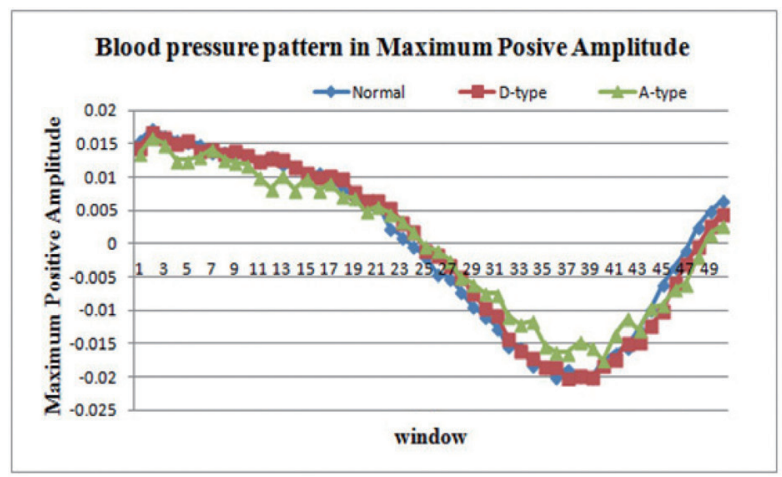

(a)

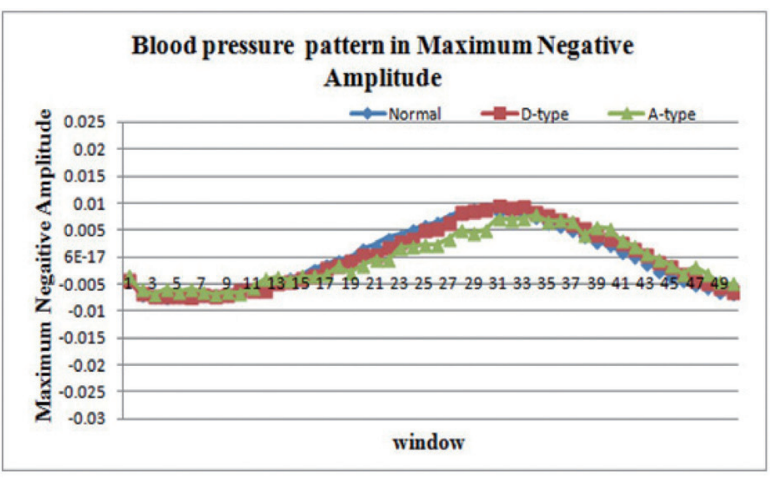

(b)

Fig. 4. MPA and MNA features averaged from three recordings in the feature windows for normal, type A, and type D personality group: (a) MPA and (b) MNA.

includes at least one heart beat in the minimal oscillation waveforms. $\Phi$ indicates the number of windows computed using these two parameters. The expression is applied as the following Eq. (2):

$$
\gamma=\min \left(X_{i j}^{N_{i}}\right), \quad \Phi=\frac{\gamma}{\delta} .
$$

As a result, a blood pressure pattern is segmented into 50 parts, and each part must include at least one heartbeat. Thus, we bounded 50 parts to comprise at least one heartbeat in the training set X. Oscillatory patterns which contain larger than the minimal number of waveforms in a blood pressure pattern specify that they have several heartbeats in a given window. A single-feature window consists of one subsection within 50 parts. Figure 3 shows two heartbeats detected within a given window. We can obtain two points of features from the oscillation amplitudes in each feature window: maximum positive amplitude (MPA) and maximum negative amplitude (MNA). The red points in Fig. 3 indicate the maximal top and bottom amplitudes obtained in the divided feature window. MPA is the amplitude of the maximal top pulse in the divided single-feature window, and MNA specifies the amplitude of the bottom pulse. Figures 4(a) 


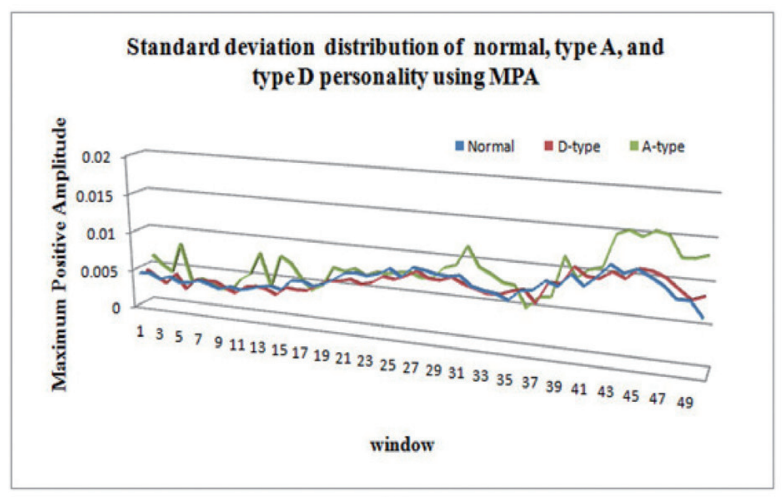

(a)

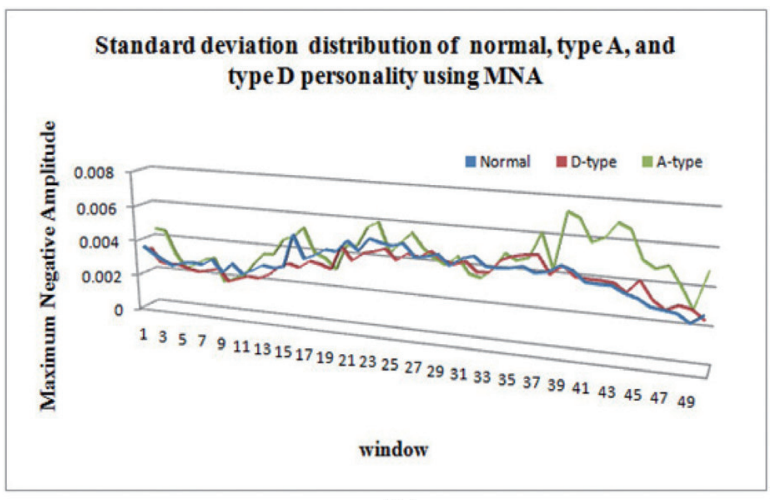

(b)

Fig. 5. Standard deviation distributions of normal, type A, and type D personality subjects using MPA and MNA: (a) MPA and (b) MNA.

and (b) describe the results of MPA and MNA feature extraction for three subject groups, which was averaged from three recordings in each window. In Figs 4(a) and (b), the average value of maximum positive and negative amplitudes did not show a large difference in the window sections between the different personality types.

Figures 5(a) and (b) display distributions of standard deviations for each three subject groups using the maximum positive and negative amplitudes. In Figs 5(a) and (b), the standard deviation distributions showed a large variance in each window section. In particular, the standard deviation distribution of type A group, compared to that of normal personalities in the MPA features in Fig. 5(a), showed a large variation in the special window section; The standard deviation distribution of type A and type D personality subjects in the MNA features in Fig. 5(b) indicated a large variation than the normal type personality subjects in each window section. These results reflect that the blood pressure patterns of type A and type D personalities, compared to normal type personalities, in the twenties age range exhibit an obvious amplitude variance between individuals, rather than uniform features. For this reason, we tried to extract the time-localized features of these amplitude variances by using a short-time Fourier transform (STFT) introduced by Gabor [20]. The expression is applied as the following Eq. (3):

$$
\operatorname{STFT}\left(t^{*}, \mu\right)=\int_{t}\left[f(t) \cdot W\left(t-t^{*}\right)\right] \cdot e^{-j 2 \pi \mu t} d t .
$$

$f(t) \cdot W\left(t-t^{*}\right)$ indicates a short-time section of the input signal $f(t)$ for each window, centered at time $t=t^{*} . W$ is a windowing function, $t^{*}$ is a time parameter, and $\mu$ is a frequency parameter. Our study used a Hamming window as a windowing function. The Hamming window size was selected by the best identification of three personality types, which was given by a sixteen feature window width in 50 window sections. Figure 6 shows the results of feature extraction for normal, type A, and type D groups using STFT based on MPA and MNA features averaged. Figure 6 displays quite different result between the three personality types. In particular, while the normal group was the most distinct when the STFT was applied to MPA features, the type A and D group was the most distinct when the STFT was applied to MNA features. Figure 7 shows the averaged STFT results among the three personality types based on MPA, MNA, and the combination of MPA and MNA features. In Fig. 7, type A and type D subjects display a fairly large frequency response difference in the $0.71 \mathrm{~dB} \sim 6.78 \mathrm{~dB}$ compared to normal 


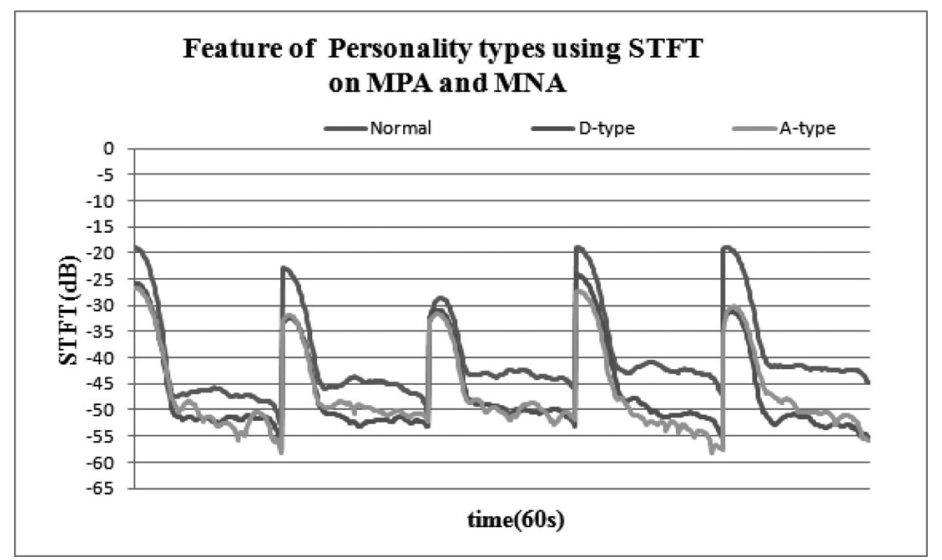

Fig. 6. Features extracted from the normal, type A, and type D personality subjects, using STFT on averaged MPA and MNA.

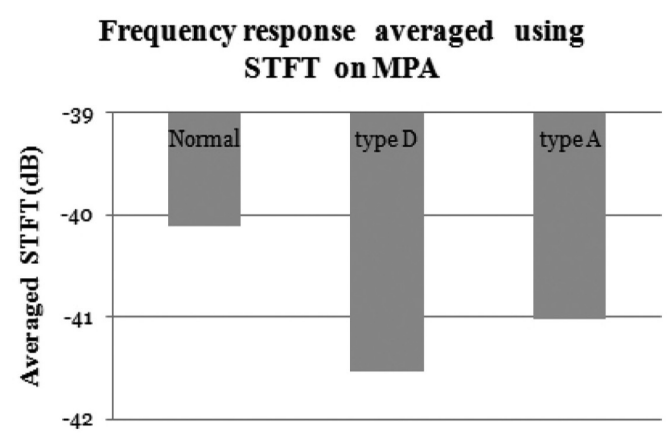

(a)
Frequency response averaged using STFT on MNA

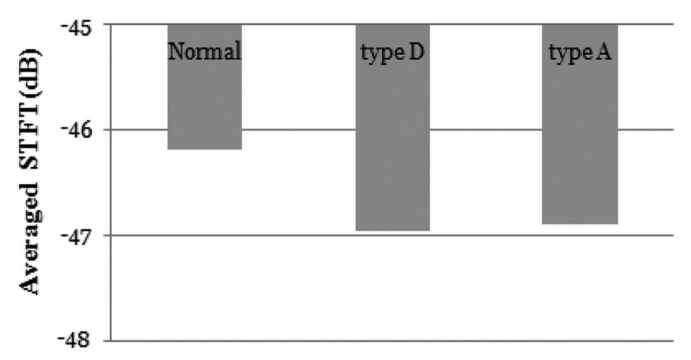

(b)

Frequency response averaged using STFT on MPA and MNA

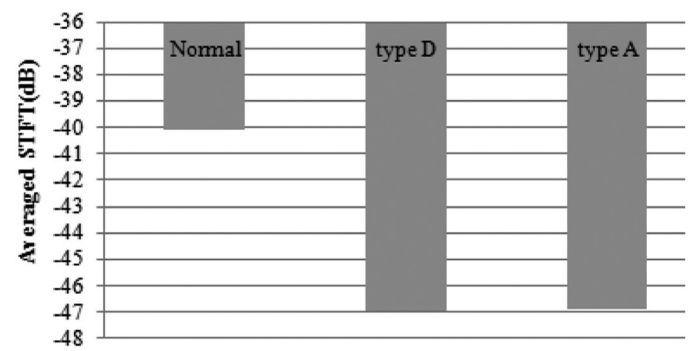

(c)

Fig. 7. Averaged STFT results across the three personality groups based on MPA, MNA, and MPA + MNA features: (a) averaged STFT based on MPA features, (b) averaged STFT based on MNA features, and (c) averaged STFT based on combination of MPA and MNA features.

personality subjects. In particular, the maximum frequency response difference, $6.78 \mathrm{~dB}$, displayed by Fig. 7(c) that indicates the averaged STFT result in Fig. 6. The type A and type D subjects exhibited frequency response differences in the $0.06 \mathrm{~dB} \sim 0.51 \mathrm{~dB}$ range. In Figs 7 (a)-(c), type A and type D subjects commonly exhibited a low-frequency response compared to that of the normals. In addition, type D subjects showed a lower frequency response differences than type A subjects. 


\subsection{Identification}

Features extracted using STFT based on MPA and MNA features, normalized in oscillometric measurements, are classified in three personality types using linear discriminant analysis (LDA). LDA is a method for searching feature vectors in the base space that could optimally discriminate between groups [21]. LDA can be summarized as a technique for reducing the dimensions of feature vectors of searching optimal categorizing vectors by maximizing the between-class scatter and minimizing withinclass scatter. The main purpose of LDA is maximization of the separation power between classes. To best identify personality types, we need to set ideal criteria for the projected data.

Given a training set $X=\left\{S T F T_{i}\right\}_{i=1}^{C}$, having $C$ classes including each class $S T F T_{i}=\left\{S T F T_{i j}\right\}_{j=1}^{C_{i}}$, containing features $S T F T_{i j}$, we have a total of $N=\sum_{i=1}^{c} C_{i}$ spectrogram patterns using STFT extracted in expression [3]. Our study defines two scales for total data set of three classes. $S_{W T}$ is described withinclass scatter matrices for feature set of the training data. $S_{B T}$ is described between-class scatter matrices for feature set of the training data. $S_{W T}$ and $S_{B T}$ are performed according to the following Eqs (4) and (5):

$$
\begin{aligned}
S_{W T} & =\frac{1}{N} \sum_{i=1}^{c} \sum_{j=1}^{c_{i}}\left(\text { STFT }_{i j}-\mu_{i}\right)\left(\text { STFT }_{i j}-\mu_{i}\right)^{T} . \\
S_{B T} & =\frac{1}{N} \sum_{i=1}^{c}\left(\mu_{i}-\mu\right)\left(\mu_{i}-\mu\right)^{T} .
\end{aligned}
$$

In Eqs (4) and (5), $S T F T_{i j}$ indicates the $j$ th feature of class $i, c$ is the total classes, $\mu_{i}$ is the average of class $i, c_{i}$ indicates features in class $i$, and $\mu$ is the average of the total classes. The LDA approach [22] detects the basis vectors represented by $\omega$ of Eq. (6) that optimizes the rate between $S_{W T}$ and $S_{B T}$.

$$
\omega=\arg \max \frac{\left|\omega^{T} S_{B T} \omega\right|}{\left|\omega^{T} S_{W T} \omega\right|}
$$

A solution supposes that $S_{W T}$ is non-singular, and that the basis vectors $\omega$ match to the first $N$ eigenvectors using the biggest eigenvalues of $S_{W T}^{-1} S_{B T}$. The LDA-based feature representation $Z=\omega^{T} S T F T$ is generated with projecting input feature set that was extracted using the short-time Fourier transform, on the subspace extended through the $N$ eigenvectors.

\section{Results}

To evaluate the performance power of our method, we carried out two experiments using STFT for the MPA and MNA features. The experimental data used 432 records collected from each of 144 subjects. The first experiment involves the train set that is used for the test set. The second experiment excludes the test set from the training set. The first experiment uses 432 records that were collected from 144 participants for the training data, which are used equally for the test set. The goal of the first experiment, Experiment I, is to evaluate the performance of clustering power for the training set. In the second experiment, Experiment II, the training data included 258 records from 86 subjects. We used remaining 174 data recordings as a testing set collected from 58 subjects, who was excluded from the training set. In Experiment II, we applied two folds for training and the remaining one for testing in the 2-fold cross 
Table 1

Subset of features extracted using the STFT, based on MPA, MNA, and MPA + MNA

\begin{tabular}{|c|c|c|c|}
\hline Subset & & & \\
\hline STFT & using maximum po & itive amp & litude (MPA) \\
\hline STFT & using maximum ne & gative amp & plitude (MNA) \\
\hline STFT & using MPA + MN & & \\
\hline & Table 2 & & \\
\hline & perimental results $b$ & ised on LI & \\
\hline Experiment & $\begin{array}{l}\text { Personality } \\
\text { type }\end{array}$ & $\begin{array}{l}\text { Applied } \\
\text { subset }\end{array}$ & $\begin{array}{l}\text { Recognition- } \\
\text { rate }(\%)\end{array}$ \\
\hline Experiment 1 & $\begin{array}{l}\text { Normal, type A } \\
\text { and type D }\end{array}$ & I & $58.8 \%$ \\
\hline & $\begin{array}{l}\text { Normal, type A } \\
\text { and type D }\end{array}$ & II & $66.2 \%$ \\
\hline & Normal & III & \\
\hline & type A and type D & II & $91.0 \%$ \\
\hline Experiment II & Normal & III & \\
\hline & type A and type D & II & $88.8 \%$ \\
\hline
\end{tabular}

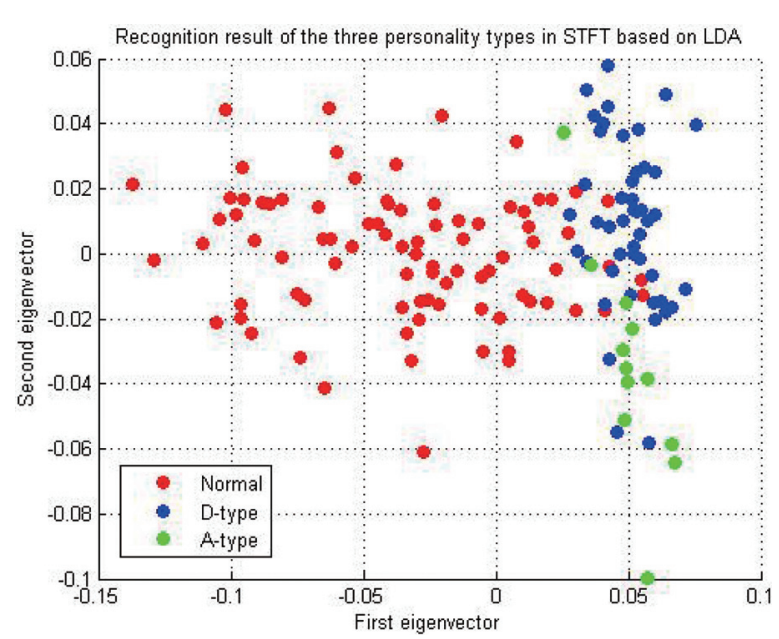

Fig. 8. Recognition results of the three personality types in STFT, based on Fig. 7(c).

validation method. The final recognition performance was estimated as the average recognition rate of each of two experiments. The goal of the Experiment II is to evaluate a more generalized performance of our approach.

The results of these two experiments were evaluated against the recognition achievement of LDA by the Mahalanobis distance. The Mahalanobis distance provides one good method of measuring similarities. To find the best fit features by LDA, we applied the three feature sets for the testing of their discrimination power. Subset I included the STFT using the maximum positive amplitude features and Subset II included the STFT using the maximum negative amplitude features. This result is described in Table 1. Subset III concatenated the MPA and MNA features into one vector. Figure 6 shows the results of feature extraction acquired from Subset III of Table 1.

The results in Fig. 7 show the frequency responses of the averaged STFT among the three personality types using the features of Subset I, Subset II, and Subset III, respectively. Figure 7(a) shows the frequency responses of the averaged STFT using Subset I for all three personality types. Figure 7(b) shows the frequency responses of the averaged STFT using Subset II for all three personality types. However, Fig. 7(c) shows very special result: The normal personality subjects in Fig. 7(c) exhibit the frequency responses of the averaged STFT using Subset III and the type A and type D personality subjects exhibit the frequency responses of the averaged STFT using Subset II. In particular, Fig. 7(c) showed the highest frequency response difference $(6.78 \mathrm{~dB})$ between the type $\mathrm{D}$ and normal personalities.

Table 2 shows the results of recognition using LDA, which yields the best identification rate among the three personality types in Fig. 7. We achieved our best personality type identification rate (91\%), using the first two eigenvectors with a combination of Subsets II and III, in Experiment I of Table 2. Figure 8 shows the recognition results of the three personality types in given the best identification rate of $91 \%$. The $x$-axis and $y$-axis of Fig. 8 display the first two eigenvectors with the largest eigenvalues of $S_{W T}^{-1} S_{B T}$, projecting the largest mean differences between the three classes. In Experiment II, each recognition result of two experiments with 2 -fold cross validation method showed $87.9 \%$ and $89.6 \%$. The average recognition rate got an identification rate of $88.8 \%$. The best identification rate in two experiments by 2 -fold cross validation method showed $89.6 \%$. The sensitivity and specificity for each class in the best recognition rate indicates in Table 3. 
Table 3

Sensitivity and specificity for each class in the best recognition rate

\begin{tabular}{|c|c|c|c|c|c|c|}
\hline \multirow{8}{*}{$\begin{array}{l}\text { Test result of three } \\
\text { types of personality }\end{array}$} & \multirow[t]{2}{*}{ Personality type } & & \multicolumn{2}{|c|}{ Actual personality type } & \multirow{2}{*}{$\begin{array}{c}\text { Sensitivity } \\
\text { (true positive) }\end{array}$} & \multirow{2}{*}{$\begin{array}{c}\text { Specificity } \\
\text { (true negative) }\end{array}$} \\
\hline & & & Yes & No & & \\
\hline & \multirow[t]{2}{*}{ Normal } & Yes & 35 & 2 & \multirow[t]{2}{*}{$94.6 \%$} & \multirow[t]{2}{*}{$90.5 \%$} \\
\hline & & No & 2 & 19 & & \\
\hline & \multirow[t]{2}{*}{ Type D } & Yes & 12 & 1 & \multirow[t]{2}{*}{$80 \%$} & \multirow[t]{2}{*}{$97.7 \%$} \\
\hline & & No & 3 & 42 & & \\
\hline & \multirow[t]{2}{*}{ Type A } & Yes & 5 & 3 & \multirow[t]{2}{*}{$83.3 \%$} & \multirow[t]{2}{*}{$94.2 \%$} \\
\hline & & No & 1 & 49 & & \\
\hline
\end{tabular}

\section{Discussion and conclusion}

This study explored the possibility of differentiating between normal and type A or type D personalities, which is reported as risk factors of cardiovascular disease. With the STFT using the combination of MPA and MNA features, we could successfully discriminate the heart disease-prone personality groups (type A and type D) from normal groups. Our best recognition results showed recognition rate of $91 \%$ in Experiment I and 88.8\% in Experiment II. This means that the our new method of analyzing blood pressure features can be a useful and noninvasive instrument in differentiating high risk group of cardiovascular disease (type A or type D personalities) from normal group.

Experiment I used the training set as a test data and showed a 9\% error rate. This error rate might have resulted from different measuring methods. That is, while we identified type A and type D with selfreporting method, the predicting variable was measured by objective biosignal measurement method. The STFT features consist of frequency responses and showed different frequency responses among the three personality types. The type A and type D personality subjects displayed a fairly lower frequency response (in the range of $0.71 \sim 6.78$ ) $\mathrm{dB}$ than the normal personality subjects. In addition, the distinction between type $\mathrm{A}$ and type $\mathrm{D}$ personalities indicated a small frequency response difference in the range of $0.06 \sim 0.51 \mathrm{~dB}$ compared to those of the normal personality. Notably, the type $\mathrm{D}$ personality exhibited the lowest-frequency response of all the three personality types. For this reason, we propose that frequency response magnitude can support a good discrimination power parameter for differentiating between normal and heart disease-prone personality types (type A and type D).

Ko and Kim [23] investigated the responses of the autonomic nervous system of individuals with type D personalities during an acute stressful situation. They reported that the type D group showed lower high-frequency activation and higher low-frequency activation than the non-type D group. This report shows the similar result to those of our type D subjects, who were activated at a lower frequency than the normal personality group. In particular, the STFT using combination of the MPA and MNA features showed the maximum low-frequency response difference of $6.78 \mathrm{~dB}$. These features enhance recognition performance. However, the combination effect of the MPA and MNA features differs with each personality type. While the type A and type D personality groups showed best recognition power only in the MNA, the normal personality group showed best recognition power with combination of the MPA and MNA. From these results, we suggest that the MNA feature can successfully represent the differentiation between type $A$ and type $\mathrm{D}$ personalities. This author [16] already proposed a new method for hypertension diagnosis using oscillometric blood pressure measurement in 2011. The research results showed that the MNA features clearly indicated the character of hypertensive or prehypertensive subjects based on systolic blood pressure. Combining with the published results, we can arrive at a probability that the STFT using the MNA features obtained from oscillometric blood pressure measurements can provide 
verification data to examine the correlation between type A and type D personalities and cardiovascular diseases.

Studies about the relationship between types A or D and heart disease have produced somewhat inconsistent results until now. The personality concept of type A or type D, a psychological risk factor of heart disease, has been measured by self-reporting method. And the method of measuring blood pressure for studying this topic has long been dependent upon two-point (systolic and diastolic). One of the main reasons of these inconsistent results of many researches in this field may be attributable to the measuring method. Our approach offers a new qualitative and noninvasive method for measuring different biological indices between normal and type A or type D personalities. When the validity of our method can be strengthened by future studies using various subject groups including clinical samples, this method will be wildly used for prescreening high risk person of cardiovascular heart disease.

\section{Acknowledgement}

This study was supported by research funds from Chosun University, 2013.

\section{References}

[1] Friedman M, Rosenman RH. Association of specific overt behavior pattern with blood and cardiovascular findings; blood cholesterol level, blood clotting time, incidence of arcus senilis, and clinical coronary artery disease. J Am Med Assoc 1959; 169: 1286-1296.

[2] Denollet J. Personality and coronary heart disease: the type-D, scale-16 (DS16). Ann Behav Med 1998; 20: 209-215.

[3] Friedman M, Thoresen CE, Gill JJ, Ulmer D, Powell LH, Price VA et al. Alteration of Type A beavior and its effect on cardiac recurrences in post myocardial infarction patients: Summary results of the recurrent coronary prevention project. Am Heart J 1986; 112: 653-665.

[4] Matthews KA. Psychological perspectives on the Type A behavior pattern. Psychol Bull 1982; 91: 293-323.

[5] Dembroski TM, Costa PT. Coronary prone behavior: components of the Type A pattern and hostility. J Pers 1987; 55: 211-235.

[6] Friedman HS, Booth-Kewley S. The "disease-prone personality": A meta-analytic view of the construct. Am Psychol 1987; 42(6): 539-555.

[7] Hemingway H, Marmot M, Evidence based cardiology: psychosocial factors in the aetiology and prognosis of coronary heart disease, Systematic review of prospective cohort studies. Br Med J 1999; 318(7196): 1460-1467.

[8] Denollet J. DS14: Standard assessment of negative affectivity, social inhibition, and Type D personality. Psychosom Med. 2005; 67: 89-97.

[9] Yaghouby F, Ayatollahi A, Soleimani R. Classification of cardiac abnormalities using reduced features of heart rate variability signal. W Appl Sci J 2009; 6(11): 1547-1554.

[10] Yaghouby F, Ayatollahi A. An arrhythmia classification method based on selected features of heart rate variability signal and support vector machine-based classifier. WC 2009, IFMBE Proceedings. 2009; 25/IV: 1928-1932.

[11] Yaghouby F, Ayatollahi A, Bahramali R, Yaghouby M, Alavi AH. Towards automatic detection of atrial fibrillation: A hybrid computational approach. Comput Biol Med 2010; 40(11-12): 919-930. doi:10.1016/j.compbiomed.2010.10.004.

[12] Yaghouby F, Ayatollahi A, Bahramali R, Yaghouby M. Robust genetic programming-based detection of atrial fibrillation using RR Intervals. Expert Sys 2012; 29(2): 183-199. doi:10.1111/j.1468-0394.2010.00571.x.

[13] South Korea. 2012 National health statistics. Government of South Korea: Ministry of Health and welfare; 2014.

[14] Dutton EP. Heart-book: a guide to prevention and treatment of cardiovascular disease. New York: The American heart association; 1980.

[15] Kaplan NM. Clinical hypertension. $2^{\text {nd }}$ ed. Baltimore: Williams and Wilkins; 1978.

[16] Shin YS. Estimation of blood pressure measurements for hypertension diagnosis using oscillometric method. IEICE Transactions 2011; E94-A (2): 806-812.

[17] Shin YS. Monitoring personalized trait using oscillometric arterial blood pressure measurements. J Appl Math 2012; 2012: 1-12. doi:10.1155/2012/591252.

[18] Kim JS, Han KH, Lim JY, Lee JH, Min BB, Moon KJ. MMPI-2: Manual for Korean version of MMPI-2, Seoul: Maumsarang Ltd; 2005. 
[19] Lee MS, Park YM, Lim HE, Song WH, Ahn JC, Kim SH et al. Preliminary study on the standardization of Korean version of type-D personality scale 14: internal consistency and construct validity. Korean J Psychosom Med 2007; 15: 81-87.

[20] Gabor D. Theory of communication. J IEE 1946; 93: 429-457.

[21] Fukunaha K. Introduction to statistical pattern recognition. $2^{\text {nd }}$ ed. Academic Press; 1990.

[22] Belhumeour PN, Hespanha JP, Kriegman DJ. Eigenfaces vs. Fisherfaces: recognition using class specific linear projection. IEEE Trans Pattern Anal Mach Intell 1997; 19: 711-720.

[23] Ko SY, Kim MS. Automatic response of female college students with Type D personality during an acute stress task: heart rate variability. Korean J Health Psychology 2009; 14: 277-292. 